\title{
An interactive fuzzy physical programming for solving multi-objective skip entry problem
}

\author{
Runqi Chai, Student Member, IEEE, Al Savvaris, Member, IEEE, Antonios Tsourdos, Member, IEEE, and Yuanqing \\ Xia, Senior Member, IEEE,
}

\begin{abstract}
The multi-criteria trajectory planning for Space Manoeuvre Vehicle (SMV) is recognised as a challenging problem. Because of the nonlinearity and uncertainty in the dynamic model and even the objectives, it is hard for decision makers to balance all of the preference indices without violating strict path and box constraints. In this paper, to provide the designer an effective method and solve the trajectory hopping problem, an Interactive Fuzzy Physical Programming (IFPP) algorithm is introduced. A new multi-objective SMV optimal control problem is formulated and parameterized using an adaptive technique. By using the density function, the oscillations of the trajectory can be captured effectively. In addition, an interactive decision-making strategy is applied to modify the current designer's preferences during optimization process. Two realistic decision-making scenarios are conducted by using the proposed algorithm; Simulation results indicated that without driving objective functions out of the tolerable region, the proposed approach can have better performance in terms of the satisfactory degree compared with other approaches like traditional weighted-sum method, Goal Programming (GP) and fuzzy goal programming (FGP). Also, the results can satisfy the current preferences given by the decision makers. Therefore, The method is potentially feasible for solving multi-criteria SMV trajectory planning problems.
\end{abstract}

\section{Index Terms}

Space Manoeuvre vehicle, multi-objective, interactive fuzzy physical programming, density function, satisfactory degree, trajectory planning.

\section{INTRODUCTION}

$\mathbf{O}$ bviously, the Space Manoeuvre Vehicles (SMV) is going to play an increasingly important role in future exploration of space. Therefore, a well-designed trajectory, particularly in the skip entry phase, is key to the stable flight and improved guidance control of the vehicle [1]-[4]. Unlike traditional mission profiles discussed in [5]-[8], the mission scenario investigated in this paper focuses on the atmospheric skip hopping, targeting the entry into the atmosphere down to a predetermined position (predetermined altitude given by the industrial sponsor of this project) and the required controls involved in returning back to the orbit. Studies can be found in the literature regarding the skip reentry of deep-space spacecraft with high speed over first cosmic velocity. However in the scenario considered in this paper, a high thrust engine would be necessary for SMV to return to low earth orbit. The authors appreciate the total amount of thrust/energy required will be too high and not practical for real implementation on today's space reentry vehicles. However, the aim was to develop methods for use on new generation of SMV that will be developed over the next 2 or 3 decades, which are reflected by the specific conditions given by the industrial partner.

The two essential functions of the trajectory planning in the skip phase are to design the trajectory and use it to provide guidance command references [9], [10]. Given the critical importance of the skip hopping trajectory, it was realized that the skip trajectory should be designed with a high efficiency and accurate way. Due to the high nonlinear nature of the problem, numerical methods are usually employed to solve these types of problems. Numerical methods for solving optimal control

R. Chai, A. Savvaris and A. Tsourdos are with the School of Aerospace, Transport and Manufacturing, Cranfield University, UK, e-mail: (r.chai@cranfield.ac.uk), (a.savvaris@cranfield.ac.uk), and (a.tsourdos@cranfield.ac.uk).

Y. Xia is with the school of Automation, Beijing Institute of Technology, Beijing, China, e-mail: (xia_yuanqing@bit.edu.cn). 
problems are divided into two major classes: indirect methods and direct methods. It is usually hard to apply the indirect method since the Hamiltonian Boundary-Value Problem (HBVP) should be derived from the original problem formulation. Therefore, this research mainly focuses on the direct method. In a direct method, the continuous-time optimal control problem is transcribed to a Nonlinear Programming problem (NLP) [11]-[15]. The accuracy and efficiency of the direct methods depend largely on the mesh refinement process. Several mesh refinement techniques are proposed in [16]-[18], illustrating the advantages of such algorithms. When the solution of the SMV trajectory hopping problem exhibits discontinuities in the state and control or their higher order derivatives, a locally dense mesh grid is typically necessary to achieve better resolution. Hence, one aspect of the investigation into an adaptive technique is designed in this work to capture accurate estimation of the location of the discontinuity.

Generally, the traditional design of entry trajectory usually targets a single objective, such as maximizing the cross range, minimizing the aerodynamic heating, etc. However, for the mission considered in this paper, the expectations for enhancing performance and saving cost are of significant importance. Therefore, it is desired to have a multi-objective SMV optimization model with several criteria varying in tolerable regions. In early times, weight-sum method is widely used to convert multiobjective formulation into traditional single objective problem. However, the determination of weight parameters is difficult and tends to be sensitive with respect to the solution. In addition, Chen et al. [19] adopt an evolutionary algorithm to transform a two-objective optimization formulation. However, the computational burden tends to be heavy and the preference of different objectives cannot be reflected clearly. In 2000, Messac designed a Physical Programming (PP) method to convert the objectives, which removes the information of priority and weight coefficients [20], [21]. But in practice, usually there are some fuzzy factors in the real system [22], [23]. Hence, in this paper, the preference function is modified by taking fuzzy factors into account.

In practice, due to the lack of physical understanding of the problem, it is difficult for the designers to specify preferences with respect to each objective function. Moreover, in large scale problems, it is almost impossible for the designers to know explicitly how much to change a given aspiration level in order to obtain a satisfactory solution. Considering these difficulties, an interactive decision-making procedure is embedded in the proposed fuzzy physical programming framework. In this way, the decision maker can modify the current preference during the optimization process.

Hereafter, the paper is organized as follows: In Section II, some mathematical preliminaries are provided. A new 3-DOF multi-objective nonlinear model including different types of constraints for the SMV is established in Section III. Section IV describes an adaptive shooting method used to discretize the optimal control problem. Then in Section V the Fuzzy Physical Programming (FPP) procedures of solving multi-objective SMV trajectory problem and an interactive decision-making process are detailed. Compromised solutions generated by employing the proposed Interactive Fuzzy Physical Programming (IFPP) approach for two strategic intents are given in Section VI.

\section{PREliminaries}

Some mathematical preliminaries are necessary to facilitate the presentation of the main results.

Definition 1. (Multi-objective Optimization problems): A general Multi-objective Optimization problems (MOPs) can be written as follows:

$$
\begin{array}{ll}
\text { Find design variables } & x=\left[x_{1}, x_{2}, \ldots, x_{n}\right] \\
\text { Minimize objective functions } & J(x)=\left[J_{1}, J_{2}, \ldots, J_{m}\right] \\
\text { subject to } & x_{\min } \leq x \leq x_{\max } \\
& h_{i}(x)=0 \\
& g_{j}(x) \leq 0 \\
& (i=1,2, \ldots, E) \\
& (j=1,2, \ldots, I)
\end{array}
$$

where $m$ is the number of different objective functions. $h$ and $g$ are the equality and inequality constraints, respectively. $E$ and 
$I$ are the number of equality and inequality constraints, respectively. $x_{\min }$ and $x_{\max }$ represent the lower and upper bounds of the design variables.

In contrast to Single-Objective Problems (SOPs), the goal of MOPs is to find a set of solutions known as pareto-optimal set. A solution in this set is called nondominated solution and is optimal in the sense that no other solutions are superior (better) to it in the current searching space when all objectives are considered. Consequently, the definition of pareto-optimal can be stated as follows:

Definition 2. (Pareto optimal) [24]: A point, $x^{*} \in X$, is in the sense of pareto-optimal if and only if there does not exist another point, $x \in X$, such that $J(x) \leq J\left(x^{*}\right)$, and $J_{i}<J_{i}\left(x^{*}\right)$ for at least one objective function.

According to Definition 2, for a MOP, there may no single optimal solution but rather a set of optimal trade-offs. All the pareto-optimal solutions are called the pareto-optimal set.

Another primary concept usually used in MOPs is given below:

Definition 3. (Nondominated and Dominated points) [24]: A vector of objective functions, $J\left(x^{*}\right) \in F$, is nondominated if and only if there does not exist another vector $J(x) \in F$, such that $J(x) \leq J\left(x^{*}\right)$ with at least one $J_{i}(x)<J\left(x^{*}\right)$. Otherwise, $J\left(x^{*}\right)$ is dominated by $J_{i}(x)$.

In Definition 3, $F$ stands for the feasible space. It is worth noting that the definition of pareto-optimal is similar to that of dominance. A pareto-optimal point is often considered the same as a nondominated point.

\section{MISSION PROFILE}

In this paper, the problem to be analyzed is a skip entry trajectory optimization problem. The general skip entry addressed is illustrated in Fig.1. Considering the mission of the SMV is to overfly the ground target at specific minimum altitude, the most challenging down control and up control of the skip flight will be considered.

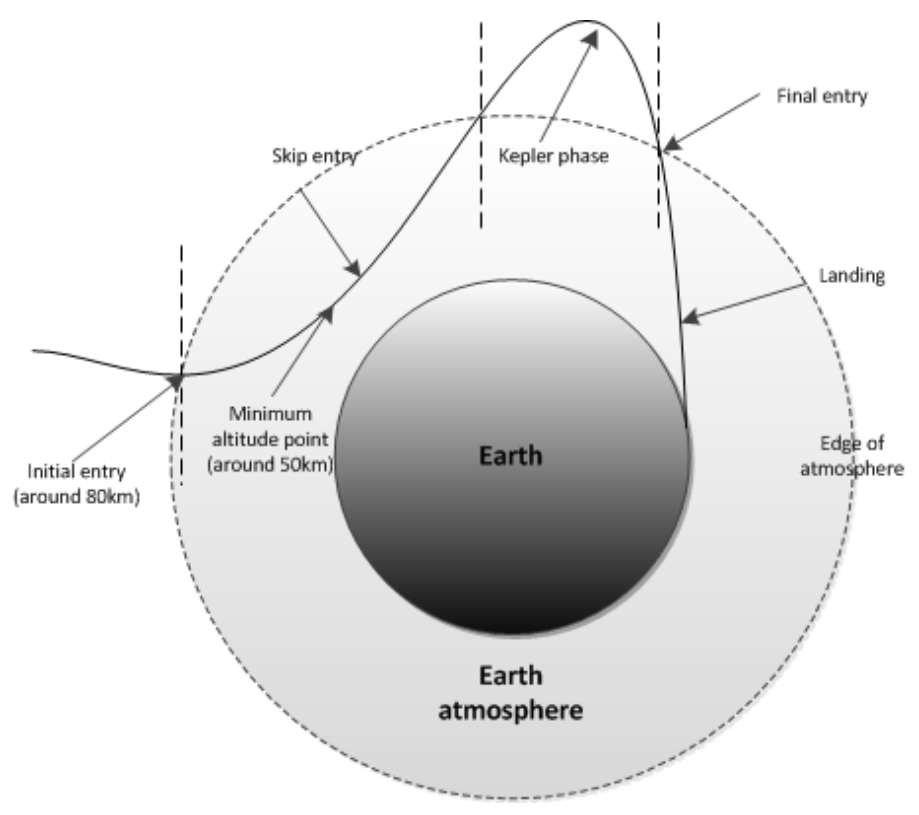

Fig. 1: General mission profile 


\section{A. Dynamics}

By considering the rotation of the Earth, the 3-DOF SMV point mass equations of motion are constructed by the following set of ordinary differential equations:

$$
\begin{aligned}
\dot{r}= & V \sin \gamma \\
\dot{\theta}= & \frac{V \cos \gamma \sin \psi}{r \cos \phi} \\
\dot{\phi}= & \frac{V \cos \gamma \cos \psi}{r} \\
\dot{V}= & \frac{T \cos \alpha-D}{m}-g \sin \gamma \\
& +\Omega^{2} r \cos \phi(\sin \gamma \cos \phi-\cos \gamma \sin \psi \cos \psi) \\
\dot{\gamma}= & \frac{L \cos \sigma+T \sin \alpha}{m V}+\left(\frac{V^{2}-g r}{r V}\right) \cos \gamma+2 \Omega \cos \phi \sin \psi \\
& +\Omega^{2} r \cos \phi(\cos \gamma \cos \phi+\sin \gamma \cos \psi \sin \phi) \\
\dot{\psi}= & \frac{L \sin \sigma}{m V \cos \gamma}+\frac{V}{r} \cos \gamma \sin \psi \tan \phi+\frac{\Omega^{2} r \cos \phi \sin \phi}{\cos \gamma} \\
& -2 \Omega(\tan \gamma \cos \psi \cos \phi-\sin \phi) \\
\dot{m}= & -\frac{T}{I_{s p} g}
\end{aligned}
$$

where $r$ is the distance from the center of the earth, $\theta$ and $\phi$ are the longitude and latitude, respectively. $V$ is the relative velocity, $\gamma$ is the flight path angle. $\psi$ is the relative velocity heading angle measured clockwise from the north, $m$ is the mass of vehicle. The control variables are angle of attack $\alpha$, bank angle $\sigma$ and thrust $T$, respectively. The earth's rotation rate is $\Omega=7.2921151 e^{-5} \mathrm{rad} / \mathrm{s}$.

The atmospheric and aerodynamic model can be summarised as:

$$
\begin{array}{ll}
g=\frac{\mu}{r^{2}} & \rho=\rho_{0} \exp \frac{r-r_{0}}{h_{s}} \\
L=\frac{1}{2} \rho V^{2} C_{L} S & D=\frac{1}{2} \rho V^{2} C_{D} S \\
C_{D}=C_{D 0}+C_{D 1} \alpha+C_{D 2} \alpha^{2} & C_{L}=C_{L 0}+C_{L 1} \alpha
\end{array}
$$

where $S=2690 \mathrm{ft}^{2}$ is reference area, $\rho$ is density of atmosphere and $\rho_{0}=0.002378 \mathrm{slug} / \mathrm{ft}^{3}$ is the density of the atmosphere at sea-level. $r_{0}=6378.135 \mathrm{~km}$ is earth radius, $L$ and $D$ are the lift and drag whereas $C_{L}$ and $C_{D}$ are the corresponding lift and drag coefficients.

In the model given by Eq.(2), three autopilot equations are introduced by using the technique of first order lag to describe the rate constraint of the controls.

$$
\begin{aligned}
\dot{\alpha} & =K_{\alpha}\left(\alpha_{c}-\alpha\right) \\
\dot{\sigma} & =K_{\sigma}\left(\sigma_{c}-\sigma\right) \\
\dot{T} & =K_{T}\left(T_{c}-T\right)
\end{aligned}
$$

Therefore, control variables are divided into actual control and demand control. The states and controls are described by:

$$
\begin{gathered}
X=[r, \theta, \phi, V, \gamma, \psi, m, \alpha, \sigma, T]^{T} \\
U=\left[\alpha_{c}, \sigma_{c}, T_{c}\right]^{T}
\end{gathered}
$$

\section{B. Box and path constraints}

SMV hopping flight should satisfy strict box and path constraints for safety reasons. Also, these conditions are depended on the mission requirements. The entire mission can be divided into two phases, the descent phase and exit phase. Due to the mission requirement, the boundary conditions for the states are:

$$
\begin{aligned}
& X=[r, \phi, \theta, V, \gamma, \psi, m, \alpha, \sigma, T]^{T} \\
& X(0)=X_{0}=\left[r_{0}, \phi_{0}, \theta_{0}, V_{0}, \gamma_{0}, \psi_{0}, m_{0}, \alpha_{0}, \sigma_{0}, T_{0}\right]^{T} \\
& {\left[r\left(t_{f 1}\right), r\left(t_{f 2}\right)\right]=\left[r_{b}, r_{f}\right]}
\end{aligned}
$$

where $t_{f 1}, t_{f 2}$ are time points for the SMV reaching the minimum altitude point and final boundary point, respectively. $r_{b}$ and $r_{f}$ are the minimum altitude point and final altitude point. During the whole time history, all the variables should satisfy the 
box constraints:

$$
\begin{array}{ll}
r_{\min } \leq r \leq r_{\max } & \theta_{\min } \leq \theta \leq \theta_{\max } \\
\phi_{\min } \leq \phi \leq \phi_{\max } & V_{\min } \leq V \leq V_{\max } \\
\gamma_{\min } \leq \gamma \leq \gamma_{\max } & \psi_{\min } \leq \psi \leq \psi_{\max } \\
\alpha_{\min } \leq \alpha \leq \alpha_{\max } & \sigma_{\min } \leq \sigma \leq \sigma_{\max } \\
m_{\min } \leq m \leq m_{\max } & T_{\min } \leq T \leq T_{\max }
\end{array}
$$

Particularly, for the demand control variables, the range should be the same with the actual controls.

$$
\begin{aligned}
& \alpha_{c(\min )} \leq \alpha_{c} \leq \alpha_{c(\max )} \\
& \sigma_{c(\min )} \leq \sigma_{c} \leq \sigma_{c(\max )} \\
& T_{c(\min )} \leq T_{c} \leq T_{c(\max )}
\end{aligned}
$$

The most important requirement for the SMV is the protection of the vehicle structure integrity, which can be achieved by setting three path constraints, aerodynamic heating, dynamic pressure and load factor.

$$
\begin{gathered}
\dot{Q}_{d}=K_{Q} \rho^{0.5} V^{3.07}\left(c_{0}+c_{1} \alpha+c_{2} \alpha^{2}+c_{3} \alpha^{3}\right)<\dot{Q}_{d \max } \\
P_{d}=\frac{1}{2} \rho V^{2}<P_{d \max } \\
n_{L}=\frac{\sqrt{L^{2}+D^{2}}}{m g}<n_{\max }
\end{gathered}
$$

where $\dot{Q}_{d \max }, P_{d \max }$ and $n_{\text {Lmax }}$ represents acceptable maximum heating rate, dynamic pressure and acceleration, respectively.

\section{Objective function}

To complete for example a reconnaissance mission in the shortest possible time interval, the first objective would be to minimize the final time. In addition, as mentioned previously, the total aerodynamic heating is very important and is also considered. On the other hand, a high final velocity will provide more kinetic energy for the vehicle. Moreover, to ensure the SMV has enough fuel to carry-out several skip hops for future exploration mission, the final objective is chosen as minimizing the fuel consumption, i.e., maximize final mass value, during the entire mission. Therefore, the four objective functions selected for the analysis are:

1). Minimizing the final time:

$$
\min J_{1}=t_{f}
$$

2). Minimizing the total aerodynamic heating:

$$
\min J_{2}=\int_{t_{0}}^{t_{f}} \dot{Q}(t) d t
$$

3). Maximizing the final velocity:

$$
\max J_{3}=V\left(t_{f}\right)
$$

4). Maximizing the final mass:

$$
\max J_{4}=m\left(t_{f}\right)
$$

\section{Relationship between different objectives}

According to the mission objectives established in Eq.(10)-(13), it is observed that the objectives in Eq.(12) and Eq.(13) are contradicting each other. More precisely, maximizing the final velocity can be achieved at the expense of fuel consumption. This can also be reflected by the velocity and mass dynamic equations. In addition, it should be noted that one of the parameters, that can increase aerodynamic heating, is dynamic pressure. Dynamic pressure is functions of density and velocity. Since density in skip entry phase is relatively small compared to the velocity, maximizing velocity in turn increases aerodynamic heating. This leads to that the objective functions in Eq.(11) and Eq.(12) are also contradicting. 
On the other hand, as can be seen from the aerodynamic heating equation, the total amount of heating is largely affected by the upper limit of integration $\left(t_{f}\right)$. Therefore, minimizing the terminal time $(10)$ and minimizing the total aerodynamic heating (11) are highly correlated objectives. Since most of the objectives are contradicting, this lead to the multi-objective design. That means a pareto-optimal solution with a good compromise between different objectives is desired for this problem. In this paper, the algorithm used to find the compromised solution is the Interactive Fuzzy Physical Programming (IFPP) approach. This algorithm uses the function transformation technique and will be further expanded in Section V.

\section{AdAPtive MUltiple SHOOTING METHOD}

To solve the continuous SMV trajectory hopping problem, parametrization algorithms should be implemented so that the continuous-time problem can be transformed to static Nonlinear Programming (NLP) problem. The discrete method used in this paper is based on multiple shooting. The basic idea of the direct multiple shooting method is to parameterize only the control variables. The controls can be approximated by interpolation at $N$ th discretized time nodes $\left[\tau_{1}, \tau_{2}, \ldots, \tau_{N}\right]$. Then the equations of motion are integrated with a fourth order Runge-Kutta method. The approximation of controls is given by:

$$
u(\tau) \approx U(\tau)=\sum_{i=1}^{N} U_{i} L_{i}(\tau)
$$

where $L_{i}(\tau)$ is a basis of Lagrange polynomials. After using direct multiple shooting method, the resulting NLP can be solved numerically by well-developed optimization algorithms such as Sequential Quadratic Programming (SQP) [25], [26] or Interior Point method (IP) [27], [28].

\section{A. Density function}

Commonly, practical guidance algorithms based on a reference trajectory which is tracked during entry phase have some degrees of model independence and do not required fast on-board computation [29]-[32], while it tends to be more sensitive in terms of initial trajectories. If the trajectory is generated on fixed distribution of mesh points, there may be some segments having a large value of violation of constraints and the accuracy of the reference trajectory is decreased. To tackle this problem, a density function technique is applied to the mesh refinement part of the direct multiple shooting process.

A mesh density function [33], [34] should be a nonnegative function $\bar{f}:[a, b] \rightarrow \Re_{+}, a, b \in \Re$ that satisfies $\int_{a}^{b} \bar{f}(t) d t=1$ and equals to zero at countably points. Since any nonnegative function $f:[a, b] \rightarrow \Re_{+}$that has only countably many zeros can be summarised as:

$$
\bar{f}(t)=\frac{f(t)}{\int_{a}^{b} f(\tau) d \tau}
$$

the corresponding cumulative distribution function $F:[a, b] \rightarrow[0,1]$ is defined as:

$$
F(t)=\int_{a}^{t} \bar{f}(\tau) d \tau
$$

The function $F$ can also be treated as a probability density function and its value corresponds to the area under the graph of $\bar{f}$ between the lower and upper bound of the integral. Clearly, $F(a)=0$ and $F(b)=1$. Consider a mesh grid $t_{i}, i=0,1,2 \ldots, N$ containing $N+1$ mesh points, and let $F$ stands for the cumulative distribution function calculated by $f$ as in Eq.(16). The position of the $(i+1)$ th point can be determined by:

$$
F\left(t_{i+1}\right)-F\left(t_{i}\right)=\frac{1}{N}
$$

Then, a mesh grid distribution can be generated based on the density function $f$ such that the distribution of grid points conforms to an equidistribution of $F$. Specifically, the mesh distribution is sparse where the value of $f(t)$ is small whereas the mesh is dense where the value of $f(t)$ is large. Note that the mesh point distribution is fixed once the density function is given. Fig. 2 illustrates 20 mesh points distribution generated by two specific density function, $f(t)=t$ and $f(t)=$ $e^{-50 t^{2}+20 t-2}+e^{-50 t^{2}+80 t-32}$, over $[0,1]$ time interval. 

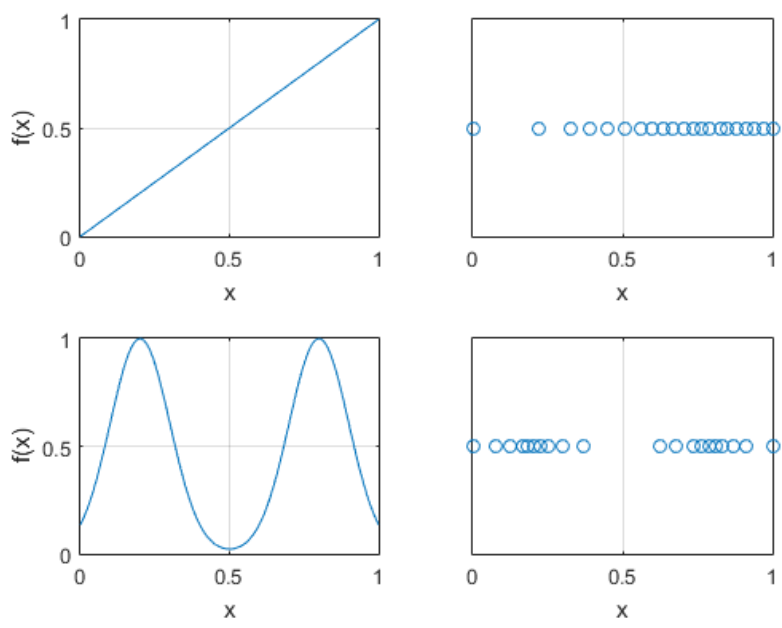

Fig. 2: Example of density function

\section{B. Adaptive multiple shooting}

In this paper, the density function based on the local curvature is used. As described in [33], assuming the number of nodes in the $j$ th iteration is $N_{j}$, the obtained state variables $\left(t_{i}, x_{i}\right)_{i=1}^{N_{j}}$ used in the $j$ th iteration are applied to calculate the curvature of the state history. To control the size of resulting NLP problem and capture the oscillation of the trajectory effectively, an adaptive multiple shooting method based on segmented density function is designed. Several points in the initial time history are selected as the segmented endpoints and the curvature equation on those subintervals can be described as:

$$
\kappa^{(k)}\left(t_{i}^{(k)}\right)=\frac{\left|\ddot{x}^{(k)}\left(t_{i}^{(k)}\right)\right|}{\left|\left(1+\dot{x}^{(k)}\left(t_{i}^{(k)}\right)\right)^{3 / 2}\right|}
$$

where $k$ is the number of subinterval. $\ddot{x}$ and $\dot{x}$ can be calculated using finite difference. Then, the segmented density function is assumed as:

$$
f^{(k)}(t)=\left(\sum_{k=1}^{m} \varrho_{(k)}^{2}(t)\right)^{\frac{1}{2}}
$$

where $\varrho_{(k)}=c^{(k)} \kappa^{(k)}(t)^{\frac{1}{3}}$ and $c$ is a constant [17]. Using the curvature information can determine whether the trajectory is flat or tends to be very noisy over the current time interval. It is possible to control the size of the resulting NLP problem by setting the number and position of segmented points. If too many points are selected, the computational time will increase, and this is seriously undesirable. On the contrary, if only one or two segmented points are selected, the accuracy of the trajectory may not be increased. Therefore, the number of selected points should be considered carefully.

\section{INTERACTIVE FUZZY PHYSICAL PROGRAMMING}

Following the discretization of the controls, the original problem has been transformed to a NLP problem with multiple objective functions described in Eq.(10) to Eq.(13). To extend the work in [35] by taking into account the decision maker's preferences during the optimization process; an interactive fuzzy physical programming algorithm is designed in this paper.

\section{A. Physical programming}

This Section briefly describes a multi-objective optimization method called physical programming (PP) [36] that is effective for generating a compromised pareto solution. The way that PP captures the decision maker's preferences is by defining preference functions. Compared with other multi-objective methods such as goal programming (GP) or evolutionary methods, the application of PP does not require the designer to specify weights for different object functions. Rather, the decision maker 
needs to define ranges of differing degrees of desirability for each objective function. In PP, different objective functions are classified into four types:

1) Class 1-S: smaller-is-better (minimization).

2) Class 2-S: larger-is-better (maximization).

3) Class 3-S: value-is-better (seek value).

4) Class 4-S: range-is-better (seek range).

Take minimization problem as an example, the boundary of the preference region is represented by some values of objective function and there are six ranges as follows:

1) Ideal range $\left(J_{i} \leq J_{i 1}\right)$

2) Desirable range $\left(J_{i 1} \leq J_{i} \leq J_{i 2}\right)$

3) Tolerable range $\left(J_{i 2} \leq J_{i} \leq J_{i 3}\right)$

4) Undesirable range $\left(J_{i 3} \leq J_{i} \leq J_{i 4}\right)$

5) Highly undesirable range $\left(J_{i 4} \leq J_{i} \leq J_{i 5}\right)$

6) Unacceptable range $\left(J_{i} \geq J_{i 5}\right)$

The components of $J_{i}$ are called the aspiration levels determined by the decision makers. Once the range parameters have been determined for each objective function, preference functions are constructed. The preference function $p_{i}\left(J_{i}(X)\right)$ are defined by using the spline interpolation.

$$
\begin{aligned}
p_{i}^{k}= & A_{0}\left(\xi_{i}^{k}\right) p_{i(k-1)}+A_{1}\left(\xi_{i}^{k}\right) p_{i k} \\
& +\bar{A}_{0}\left(\xi_{i}^{k}, \lambda_{i}^{k}\right) s_{i(k-1)}+\bar{A}_{1}\left(\xi_{i}^{k}, \lambda_{i}^{k}\right) s_{i k}
\end{aligned}
$$

where $\xi_{i}^{k}=\left(J_{i}-J_{i(k-1)}\right) /\left(J_{i k}-J_{i(k-1)}\right)$ and $0<\xi_{i}^{k}<1, \lambda_{i}^{k}=\left(J_{i k}-J_{i(k-1)}\right), k$ is the number of region and $k=2,3,4,5$. The basic functions in Eq.(20) are defined as follows:

$$
\begin{gathered}
s_{i k}=\left.\frac{\partial p_{i}^{k}}{\partial J_{i}^{k}}\right|_{J_{i}^{k}=J_{i k}} \\
A_{0}(\xi)=\frac{1}{2} \xi^{4}-\frac{1}{2}(\xi-1)^{4}-2 \xi+\frac{3}{2} \\
A_{1}(\xi)=-\frac{1}{2} \xi^{4}-\frac{1}{2}(\xi-1)^{4}+2 \xi-\frac{1}{2} \\
\bar{A}_{0}(\xi, \lambda)=\lambda\left[\frac{1}{8} \xi^{4}-\frac{3}{8}(\xi-1)^{4}-\frac{1}{2} \xi+\frac{3}{8}\right] \\
\bar{A}_{1}(\xi, \lambda)=\lambda\left[\frac{3}{8} \xi^{4}-\frac{1}{8}(\xi-1)^{4}-\frac{1}{2} \xi+\frac{1}{8}\right]
\end{gathered}
$$

\section{B. Sensitivity analysis}

Generally, the PP method involves converting different objective functions into a single function by using the preference function presented in the previous Section. Then it is solved to get a compromised pareto point. The sensitivity at the current pareto point calculated by PP can provide the variation in one objective function given the variation in another objective function on the pareto surface in a given direction. It can be treated as directional derivatives and is a tangent line to the pareto surface. At a compromised pareto optimal point $x^{*}$, assume $g\left(x^{*}\right)$ stands for the set of active constraints, which can be determined by using well-developed NLP methods. Let $C$ represent the Jacobian matrix of the active constraints at $x^{*}$. The equations of $C$ and the projection matrix $P$ of the active set are given by:

$$
\begin{gathered}
C=\nabla_{x} g\left(x^{*}\right) \\
P=I-C^{T}\left(C C^{T}\right)^{-1} C
\end{gathered}
$$


where $I$ is the identity matrix. It should be noted that, a feasible direction with the greatest improvement of objective function $J_{i}$ is achieved by projecting $\nabla_{x} J_{i}$ onto the projection matrix $P$

$$
d_{f_{i}}=P\left(-\nabla_{x} f_{i}\right) \quad i=1,2, \ldots, m
$$

where $m$ is the total number of objective function. The pareto sensitivity can be described as $d J_{i} / d J_{j}$ along the feasible descent direction of objective $J_{j}$. Then the derivatives of $J_{i}$ and $J_{j}$ with respect to optimization parameters can be rewritten as:

$$
\begin{aligned}
& d J_{i}=d_{J_{i}}^{T} d x \\
& d J_{j}=d_{J_{j}}^{T} d x
\end{aligned}
$$

Based on Eq.(29) and Eq.(30), $d x$ is solved:

$$
d x=\left[d_{J_{j}} d_{J_{j}}^{T}\right]^{-1} d_{J_{j}} d J_{j}
$$

From Eq.(31), the change in design variables $(x)$ along the feasible decent direction of objective $J_{j}$ can be calculated. Therefore, by replacing $d x$ in Eq.(30), the term $d J_{i} / d J_{j}$ can be achieved.

$$
\frac{d J_{i}}{d J_{j}}=d_{J_{i}}^{T}\left[d_{J_{j}}^{T} d_{J_{j}}\right]^{-1} d_{J_{j}} \quad i=1,2, \ldots, m
$$

Consequently, the sensitivity information can be summarised in a matrix form, called the tradeoff matrix, whose $i$ th row stands for the tradeoffs required for an improvement in the $i$ th objective function.

$$
T=\left(\begin{array}{cccc}
1 & \frac{d J_{2}}{d J_{1}} & \cdots & \frac{d J_{m}}{d J_{1}} \\
\frac{d J_{1}}{d J_{2}} & 1 & \cdots & \frac{d J_{m}}{d J_{2}} \\
\vdots & \vdots & \ddots & \vdots \\
\frac{d J_{1}}{d J_{m}} & \frac{d J_{2}}{d J_{m}} & \cdots & 1
\end{array}\right)
$$

Thus, the pareto optimality can be guaranteed only if the corresponding off-diagonal element in the matrix is negative, which means the tradeoff between two objective functions exist. On the contrary, if there is a positive element, then both of the corresponding objective can be improved at the same time.

\section{Fuzzy preference function}

By taking into account the decision maker's physical understanding of the desired design outcomes, a fuzzy preference is introduced during the optimization process. In this way, it will enable the decision maker to control the optimization to some extent. Take Class-1 as an example, the parameter $f_{i k}$ as a normal fuzzy number $\tilde{f}_{i k}$ is defined and therefore, its membership function follows the form:

$$
\mu_{\tilde{J}_{i k}}\left(J_{i}\right)=\exp \left(-\left[\frac{J_{i}-J_{i k}}{\delta_{i k}}\right]^{2}\right), \quad \delta_{i k}>0
$$

where $\delta_{i k}$ is the fuzzy parameter of the $k$ th boundary of preference function and it can be defined based on [22], [23], [35]. Then the fuzzy preference function is defined as follows:

$$
J_{p_{i k}}\left(\tilde{J}_{i k}\right)=\frac{\int_{J_{i}(X)-3 \delta_{i k}}^{J_{i}(X)+3 \delta_{i k}} \bar{J}_{i}\left(J_{i}\right) \mu_{\tilde{J}_{i k}}\left(J_{i}\right) d J_{i}}{\int_{J_{i}(X)-3 \delta_{i k}}^{J_{i}(X)+3 \delta_{i k}} \mu_{\tilde{J}_{i k}}\left(J_{i}\right) d J_{i}}
$$

where $\bar{J}_{i}\left(J_{i}\right)$ is the preference function of $i$ th objective function without considering fuzzy factor. The fuzzy preference function is shown in Fig.3. 


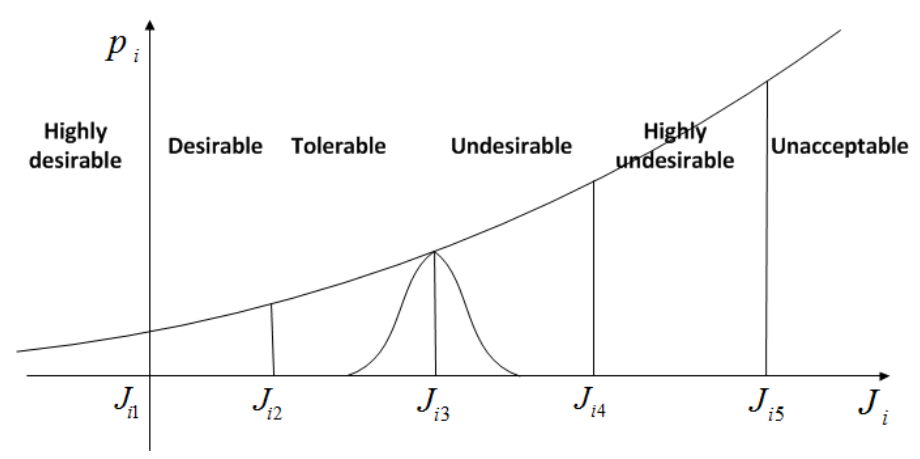

Fig. 3: Fuzzy preference function for Class 1-S

\section{Interactive fuzzy physical programming formulation}

In practice, if a solution from FPP is trusted as an approximate pareto optimal solution, there is no other process to improve all of the objective functions. Thus, if the designer wants to improve the quality of a specific objective function, it can only be achieved at the expense of other objectives. To make the proposed algorithm more controllable, an interactive process has been designed. It should be noted that, this decision making process has to be iterative and interactive since the designers may change the current preference as they know more about the current problem. The flowchart of the interactive process is illustrated in Fig.4. As can be seen from the flowchart, the approximate pareto solution is gained by solving a FPP

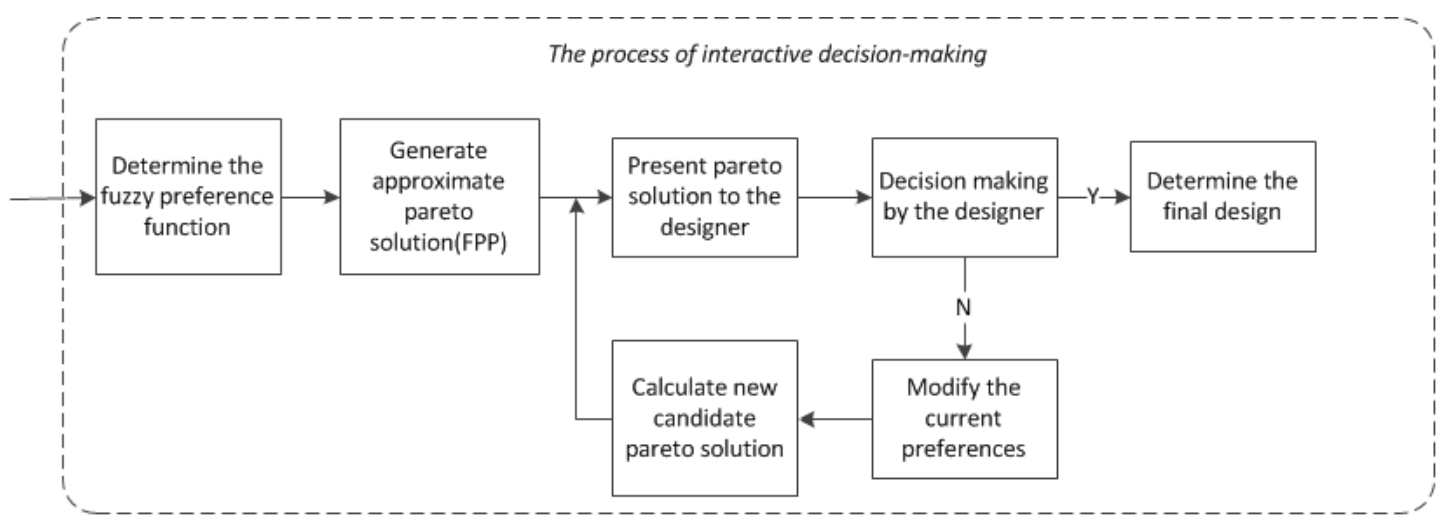

Fig. 4: Interactive decision-making procedure

model described in $\mathrm{Eq}(36)$.

$$
\begin{cases}\min P=\log _{10}\left\{\frac{1}{n_{s}} \sum_{i=1}^{n_{s}} J_{p_{i k}}\left(\tilde{J}_{i k}\right)\right\}, & \\ J_{i}(X) \leq J_{i 5}, & \text { for class 1-S; } \\ J_{i}(X) \geq J_{i 5}, & \text { for class 2-S; } \\ J_{i 5 L} \leq J_{i}(X) \geq J_{i 5 R}, & \text { for class 3-S; } \\ J_{i 5 L} \leq J_{i}(X) \geq J_{i 5 R}, & \text { for class 4-S; } \\ x_{l} \leq x \leq x_{u}, & .\end{cases}
$$

where $n_{s}$ is the number of ranges associated with the problem, $x_{l}$ and $x_{u}$ are the lower and upper bound of the design variables, respectively. For the decision-making part, the designer should determine if the approximate pareto points satisfy his/her current preferences. Once it can satisfy, the iteration will stop. Otherwise, the sensitivity of the current solution should be analyzed as it can help the decision maker gain a better understanding of relationships between each objective under current preferences. After that, if the designer decides to make a compromise between some objectives, the new approximate pareto solutions based on the updated preferences are generated such that it can satisfy the designer's current preferences. This iterative and interactive process is continued until the designer is satisfied with one of the potential solutions to be satisfactory.

The overall procedure of the designed IFPP method is detailed below: 
Step 1 Perform a fuzzification for different objectives based on Eq.(34) and Eq.(35).

Step 2 Build the preference function for each objective. The decision maker should specify the class type and region bounds for each objective function based on his/her preference.

Step 3 Construct the fuzzy physical programming model according to Eq.(36). Calculate the value of aggregate objective function without violating various constraints. Project the value in objective space and rank the alternatives in terms of the satisfactory degree.

Step 4 Visualize the obtained optimal solution. Therefore, the decision maker is able to analyze the obtained solution directly, and specify whether the current solution can meet the designer's preference. If yes, stop the procedure. Otherwise, decide which objective should be emphasized or which objective should be sacrificed.

Step 5 Specify the preference for the design objectives after investigating the obtained solution.

Step 6 Generate the preference for each objective by adjusting the value of region bounds based on the newly designed preference.

Step 7 Modify the new preference function according to the results from Step.6.

Step 8 Go to Step. 3 and use the fuzzy physical programming to solve the modified problem.

\section{Simulation RESUlTS}

\section{A. Parameter setting}

The initial and terminal conditions of the entire process and boundary constraints can be found in [35]. Only the first hop is taken into account in the paper. The initial altitude is around $80 \mathrm{~km}$, where is the assumed edge of the atmospheric layer. The drag and lift coefficients, the maximum values of heating, dynamic pressure and load factor are shown in Table I.

TABLE I: Coefficients and maximum value of path constraints

\begin{tabular}{cccc}
\hline \hline$C_{L 0}$ & $C_{L 1}$ & $C_{D 0}$ & $C_{D 1}$ \\
-0.2070 & 1.676 & 0.07854 & -0.3529 \\
\hline \hline$C_{D 2}$ & $c_{0}$ & $c_{1}$ & $c_{2}$ \\
2.040 & 1.067 & -1.101 & 0.6988 \\
\hline \hline$c_{3}$ & $Q_{d \max }$ & $P_{d \max }$ & $n_{L \max }$ \\
-0.1903 & $200 B t u / f t^{2} / s$ & $13406.46 P a$ & 2.5 \\
\hline \hline
\end{tabular}

\section{B. Pareto front generation}

In order to better show the relationship between different mission objectives (shown in Eq.(10)-(13)), a stochastic-based MOP solver, named Nondominated Sorted Genetic Algorithm-II (NSGA-II) [24], is applied to generate the approximated pareto front. These results are plotted in Fig.5-7.

As can be seen from Fig.5 to 7, the simulation results follow the analysis in Section III.D. The pareto fronts are projected onto three planes: Minimizing terminal time versus minimizing aerodynamic heating, maximizing terminal speed versus maximizing terminal mass, and minimizing aerodynamic heating versus maximizing terminal speed.

It is worth noting that the solution shown in Figs.5-7 can hardly be accepted as the accurate pareto front. This is because these solutions were carried out using a stochastic-based MOP solver. When stochastic processes are embedded in the optimization algorithm, the optimality will be affected to some extent. Moreover, since for a stochastic-based MOP solver, the solution is obtained under limited computing power, the calculated solution may deviate from the true solution. Therefore, results shown in Figs.5-7 can only be used to reflect the contradicting relationships.

Since the NSGA-II algorithm computes the entire pareto front and uses evolutionary strategies, it is usually time-consuming to calculate a high quality solution. Alternatively, the proposed IFPP method can achieve a good compromise by transforming different objectives. 


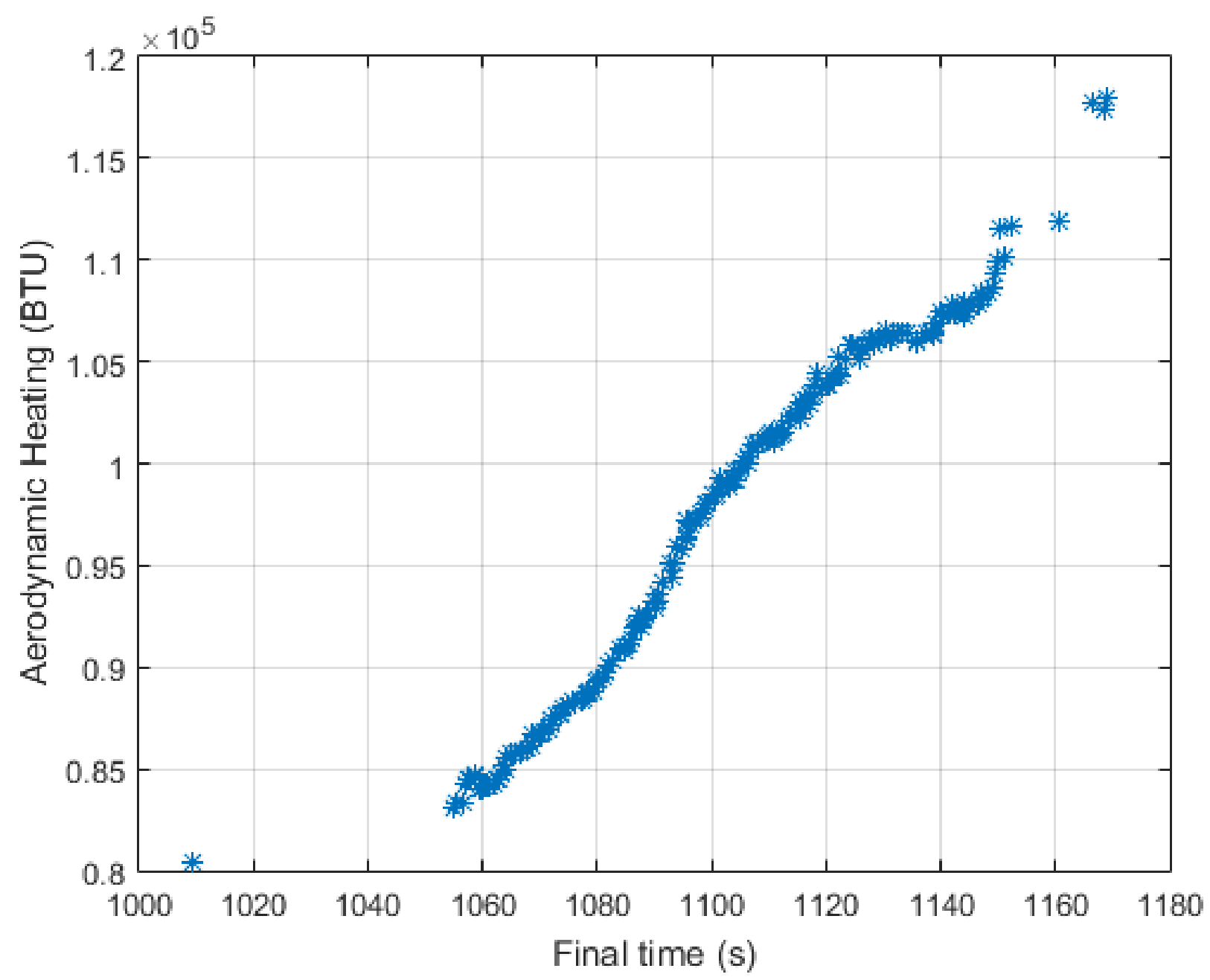

Fig. 5: Pareto front obtained via NSGA-II

\section{IFPP Results and discussion}

The initial aspiration levels for FPP are obtained by solving each corresponding single objective problem. From the single-objective solutions (as shown in Fig.8), the FPP model can be constructed as follows:

$$
\left\{\begin{array}{c}
\min \quad \log _{10}\left\{\frac{1}{5} \sum_{i=1}^{5} J_{p_{i k}}\left(\tilde{J}_{i k}\right)\right\} \\
J_{1}(x) \leq 2086.42 \\
J_{2}(x) \leq 217.63 \\
J_{3}(x) \geq 4016.27 \\
J_{4}(x) \geq 51018.23 \\
x_{l} \leq x \leq x_{u} \\
\dot{Q}_{d}=K_{Q} \rho^{0.5} V^{3.07}\left(c_{0}+c_{1} \alpha+c_{2} \alpha^{2}+c_{3} \alpha^{3}\right)<200 B T U \\
P_{d}=\frac{1}{2} \rho V^{2}<13406.4583 P a \\
n_{L}=\frac{\sqrt{L^{2}+D^{2}}}{m g}<2.5 \\
((6),(7),(8) \text { and }
\end{array}\right.
$$

To verify the effectiveness of FPP, comparative simulations have been conducted applying weighted-sum method, Goal Programming (GP) [37] and Fuzzy Goal Programming (FGP) techniques [38]. The control parameters of the weighted-sum method are shown in Table II while the results are demonstrated in Fig.9 and Fig.10 in terms of the designer's preferences 


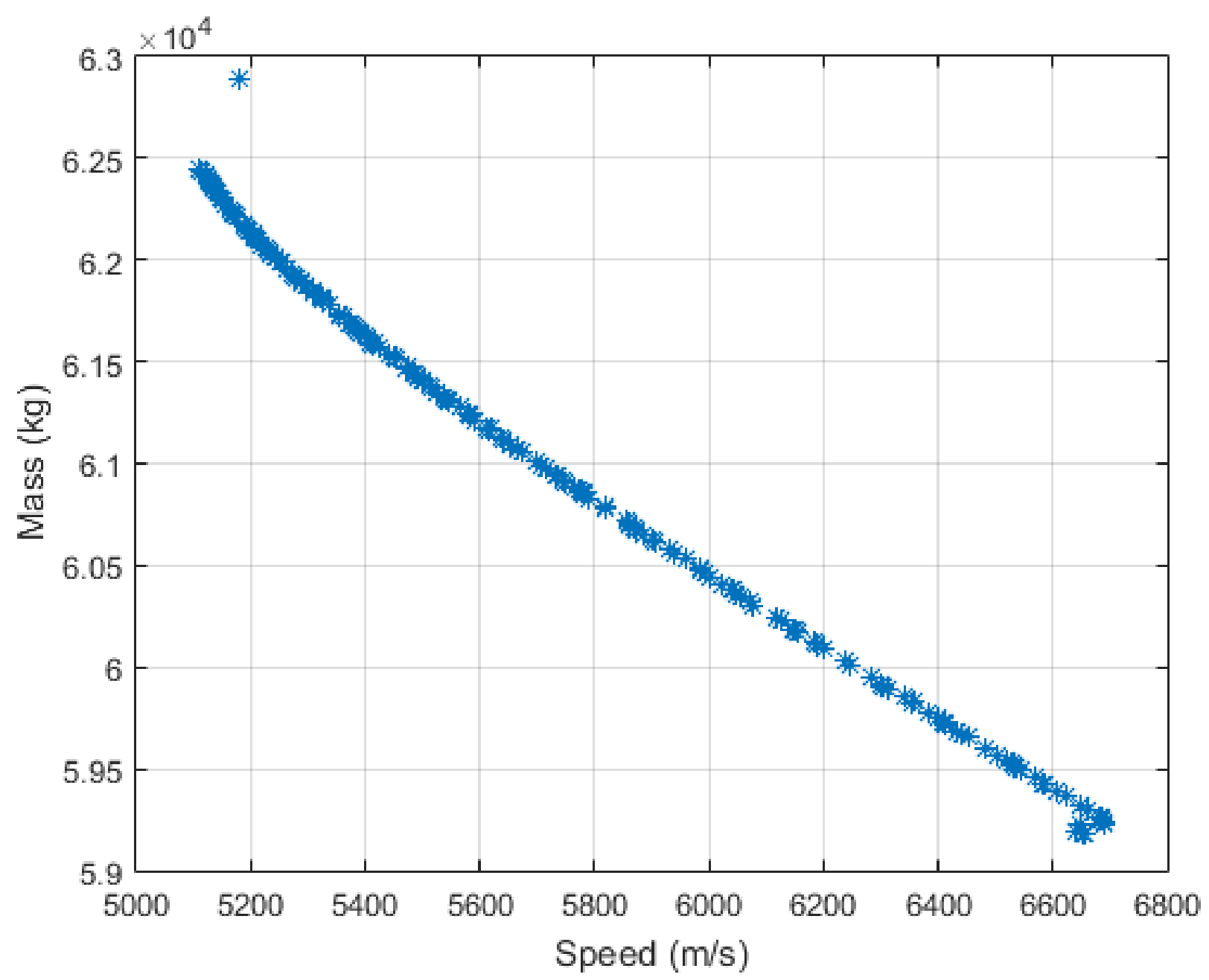

Fig. 6: Pareto front obtained via NSGA-II

in the preference space. The values of the vertical axis present the boundaries of preference function from highly desirable range to unacceptable range, which are quantified in turn as 1,2,3,4,5 by the designer. It is clear from the results that the proposed FPP has successfully derived the solutions into a tolerable or acceptable region whereas the weighted-sum, GP and FGP algorithms have failed to move all the objective functions into a tolerable region of the pareto set. This is because for goal-programming class multi-objective methods, if the designers want to specify his/her preferences in different objectives, it can only be achieved by setting additional inequality constraints. This is usually undesirable in the optimization procedure. Moreover, since the pareto tradeoff exist between different objective functions implicitly, the sensitivity analysis can be adopted at the current approximate pareto point. Based on the discussion and Eq.(33) shown in the previous section, the tradeoff table is calculated and projected in the objective space as:

$$
T=\left(\begin{array}{cccc}
1.000 & 0.474 & -0.762 & -0.889 \\
0.642 & 1.000 & -0.795 & -1.118 \\
-0.576 & -0.487 & 1.000 & -0.109 \\
-0.201 & -0.532 & -0.649 & 1.000
\end{array}\right)
$$

As can be seen from the matrix, all the non-diagonal elements in the third and fourth rows are negative. At least one element in the first and second rows is negative. These results indicate that the approximated pareto solution can be trusted. Specifically, an improvement in any objective function must associate a decrease in terms of the quality of other objective functions. This 


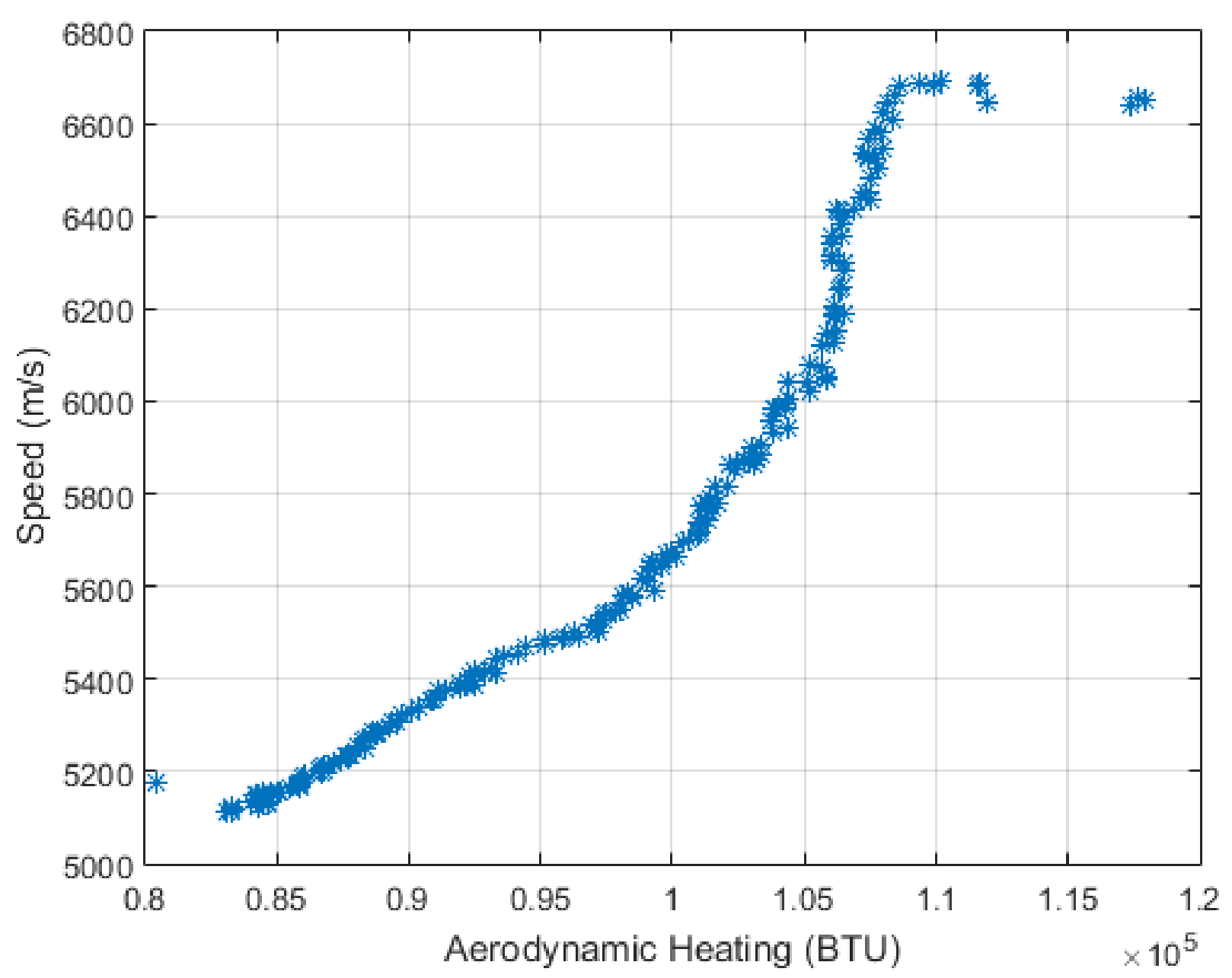

Fig. 7: Pareto front obtained via NSGA-II

is also proved numerically in [35]. Note that this criterion is only necessary but not sufficient.

TABLE II: Control parameters of weight-sum and FPP methods

\begin{tabular}{c|cc}
\hline \hline & Weights & Objective degrees $\left(J_{1}, J_{2}, J_{3}, J_{4}\right)$ \\
\hline case 1 & $(0.25,0.25,0.25,0.25)$ & $(3.25,3.41,2.42,2.21)$ \\
case 2 & $(0.40,0.20,0.20,0.20)$ & $(2.17,2.25,4.08,3.12)$ \\
case 3 & $(0.20,0.40,0.20,0.20)$ & $(2.82,2.78,3.78,2.35)$ \\
case 4 & $(0.20,0.20,0.40,0.20)$ & $(2.89,3.25,2.21,3.15)$ \\
\hline
\end{tabular}

After generating the initial solutions using FPP, the pareto data are presented to the designers in preference function space as shown in Fig.9-10. Then the interactive decision making strategy will be applied. This is the key procedure in IFPP since the decision maker may specify his/her preferences on a specific objective function based on his/her basic satisfaction to the initial candidate solutions obtained by the normative programming method. Once the decision maker specifies new preference objectives, the main process is to effectively calculate new pareto solutions, which can satisfy designer's current preference based on FPP. For the SMV skip entry mission, two scenarios of decision making are taken into account in this paper:

1. Scenario 1: The SMV needs to get the predetermined position quicker for strategic reasons.

2. Scenario 2: The SMV needs to keep more fuel so that it can do further exploration work.

It is worth noting that both the two case studies are solved by using the proposed IFPP method. These two scenarios represent two different decision maker's preferences. In Scenario 1, the goal is to improve the quality of $J_{4}$ and it is not desired 

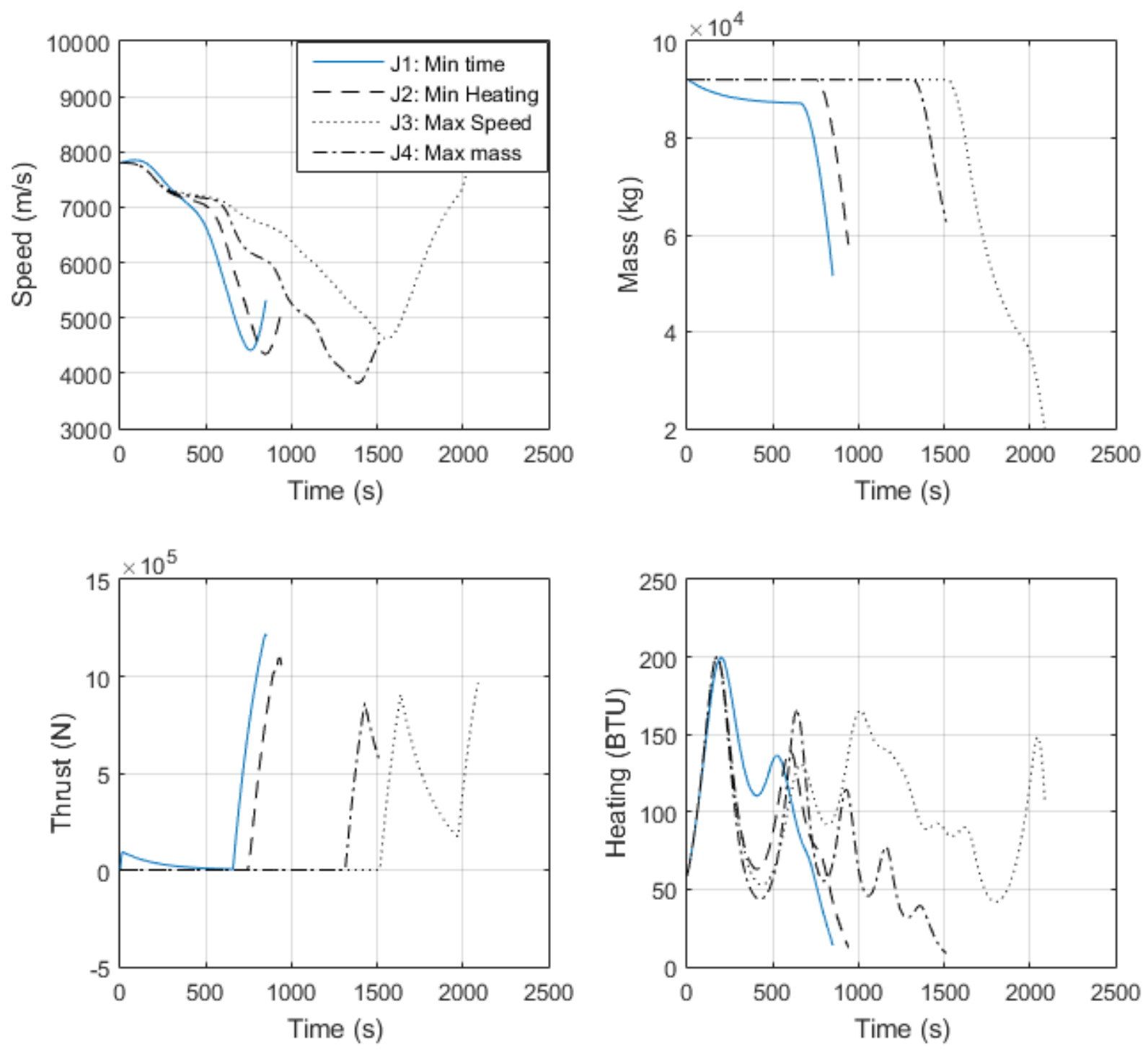

Fig. 8: Single-objective solutions

that other objective functions are sacrificed out of the tolerance regions, which means $J_{1}, J_{2}$ and $J_{3}$ can only change up to $J_{13}, J_{23}$ and $J_{33}$. Four alternative pareto solutions, which can satisfy the designer's current interest are presented in Fig.11 and the corresponding time histories in terms of the states and controls are shown in Figs.12-16. According to Figs.12-16, an attempt is made to analyze characteristic arcs of the trajectories. The trajectory is split into two subintervals: descending and climbing. In multi-objective SMV trajectory problem, aerodynamic heating tends to reach the peak value at around 200s. In general, aerodynamic heating is direct functions of angle of attack $\alpha$, dynamic pressure $P_{d}$ and lateral acceleration. It can be seen from Fig.13 that both dynamic pressure and lateral acceleration decrease significantly after 200s. Therefore, although the angle of attack is still increasing after 200s, aerodynamic heating will not surpass its peak value (200BTU) and starts to decrease. On the other hand, increasing the angle of attack will have positive influences in terms of slowing down the vehicle so that the dynamic pressure and load factor path constraints will not become active. Therefore, the structural integrity of the SMV can be protected.

After reaching the target point, the engine starts working to ensure the SMV can have enough kinetic energy to go back to the orbit. With the decrease of air density and mass, the aerodynamic heating, dynamic pressure and load factor will also 


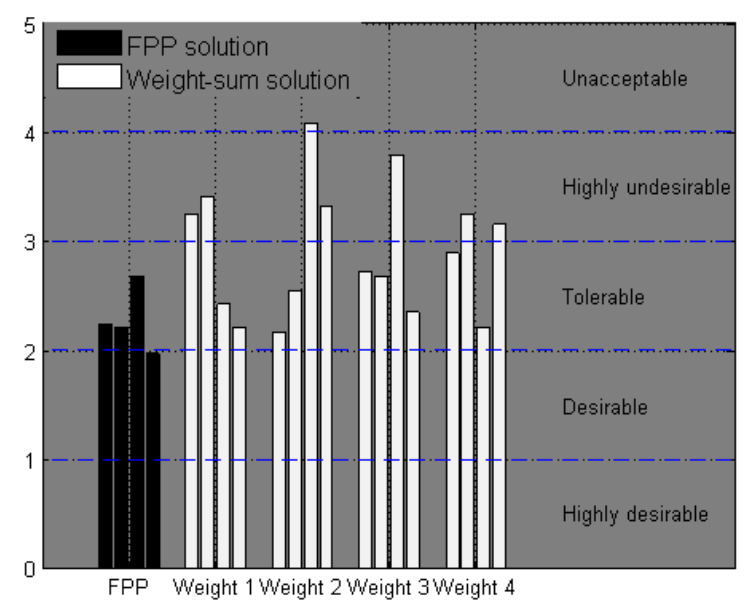

Fig. 9: Comparison between weight-sum and FPP

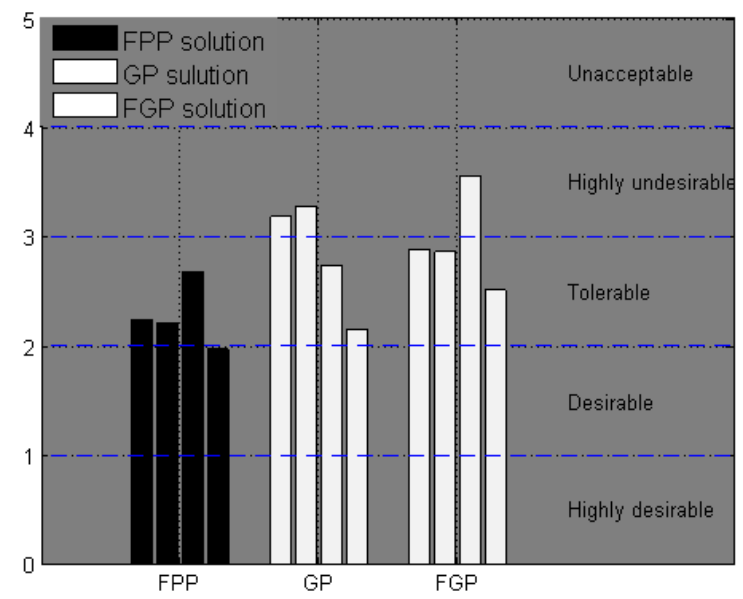

Fig. 10: Comparison between GP, FGP and FPP

decrease during the climbing phase. It should be noted that, there is a sharp decrease in the thrust curve before the SMV reaches the final boundary condition. As maximizing the final mass value is one of the objective, there must be some conflicts between maximizing final mass and other objectives, as illustrated in Eq.(38). Thus, the vehicle cannot use the maximum acceleration during the whole climbing phase. Moreover, the demand controls are demonstrated in Figs.14-16. By introducing the three autopilot equations of the control variables, the actual controls become smoother. Since the actual controls appear in the right hand side of the motion equations, the demand controls will behave in a bang-bang style as shown in Figs.14-16.

In Scenario 2, the aim of this attempt is to improve the quality of $J_{1}$ at the expanse of the other three objective functions. Similarly, four alternative solutions are generated using IFPP and projected onto the preference space illustrated in Fig.17. Compared to the initial design, it is obvious that the satisfactory degree of $J_{1}$ is improved significantly as what designer desired. However, the satisfactory degree of $J_{3}$ and $J_{4}$ becomes worse. To achieve a smaller value of final time, the engine should spend more time on keeping the SMV at maximum acceleration and because of this, the satisfactory degree of $J_{4}$ will reach its edge of tolerable region. After the decision-making process, the designer can ether accept one of the four alternative solutions as a satisfactory solution based on Fig.4 or modify the current settings and rerun the iterative process.

As for the performance of the adaptive multiple shooting method, the density function based on curvature information is used for the mesh refinement part for the SMV trajectory optimization problem. The density function is shown in Eq.(19). By 


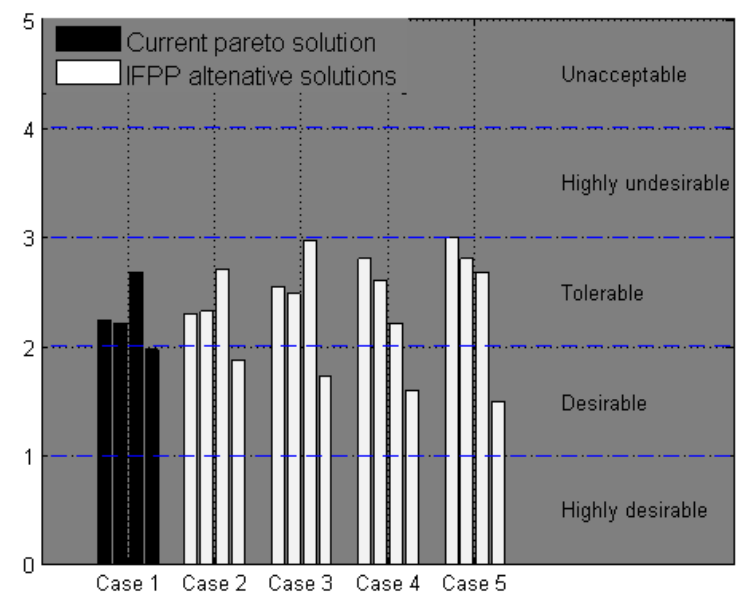

Fig. 11: Alternative solutions for Scenario 1 generated by IFPP
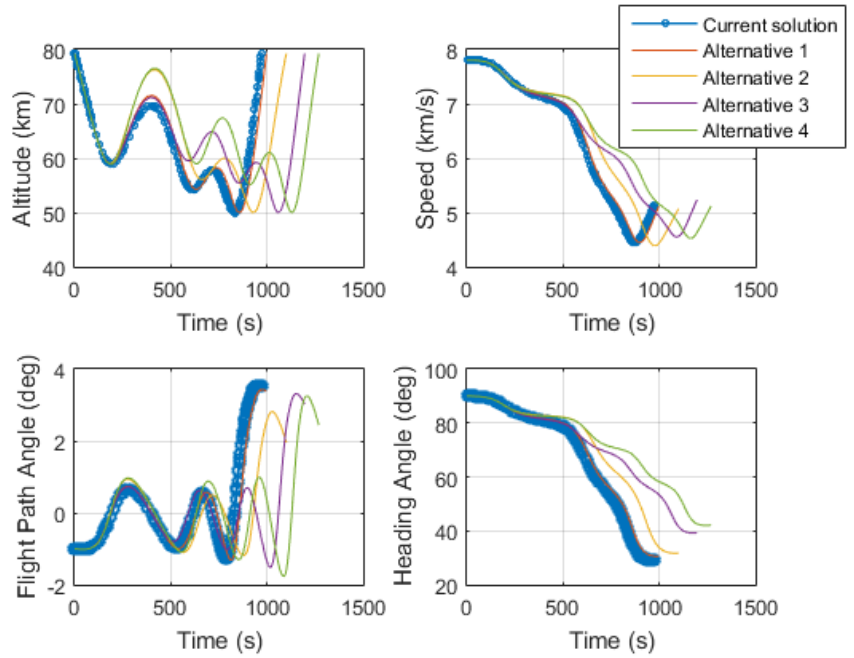

Fig. 12: Trajectories for altitude, speed, flight path angle and heading angle in five cases
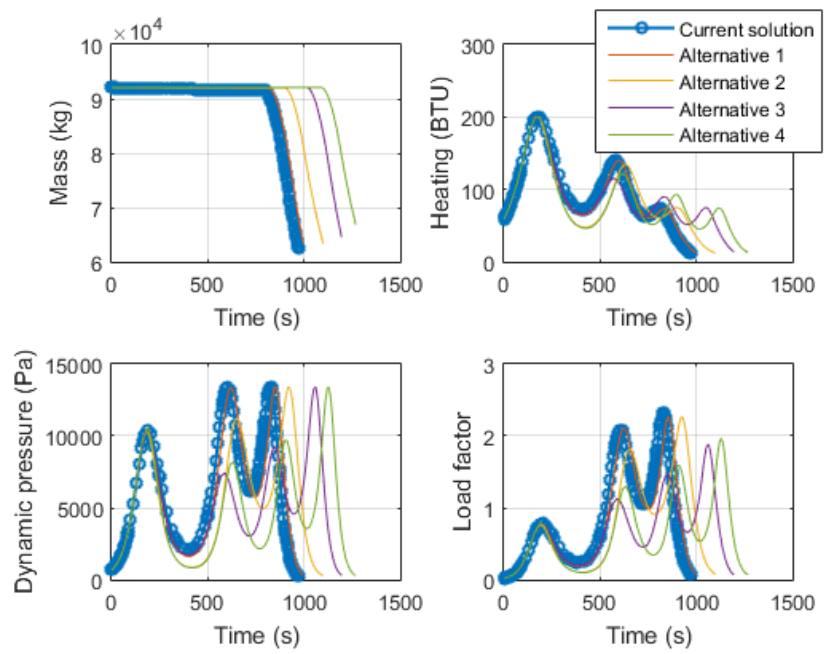

Fig. 13: Trajectories for mass and path constraints in five cases 

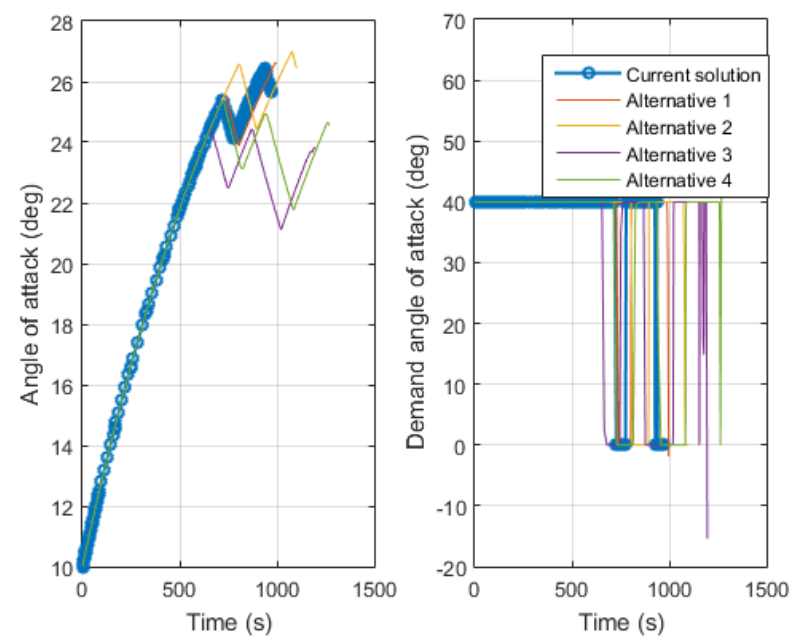

Fig. 14: Trajectories for actual AOA and demand AOA in five cases
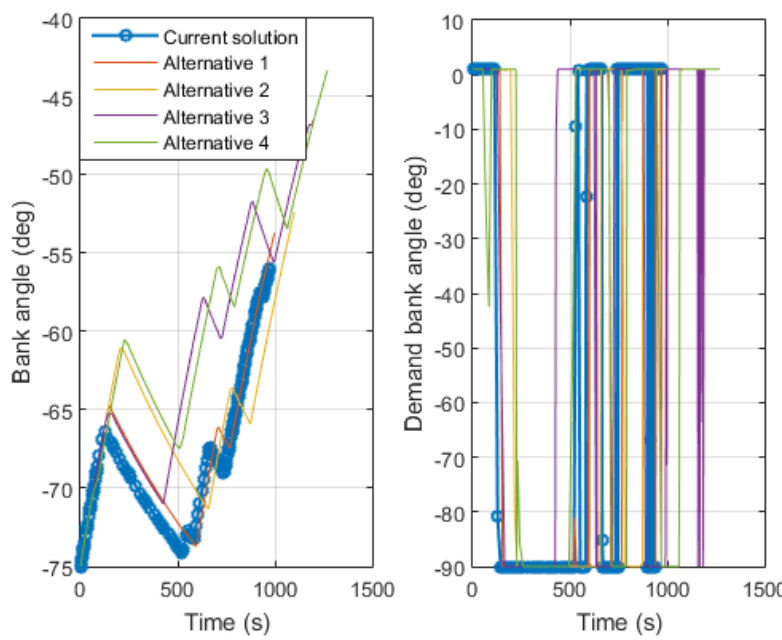

Fig. 15: Trajectories for actual bank angle and demand bank angle in five cases
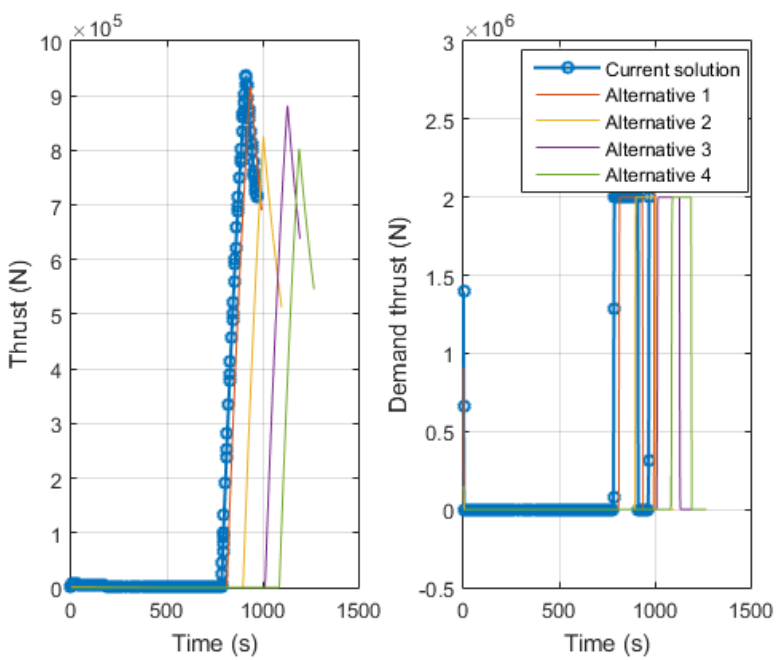

Fig. 16: Trajectories for actual thrust and demand thrust in five cases 


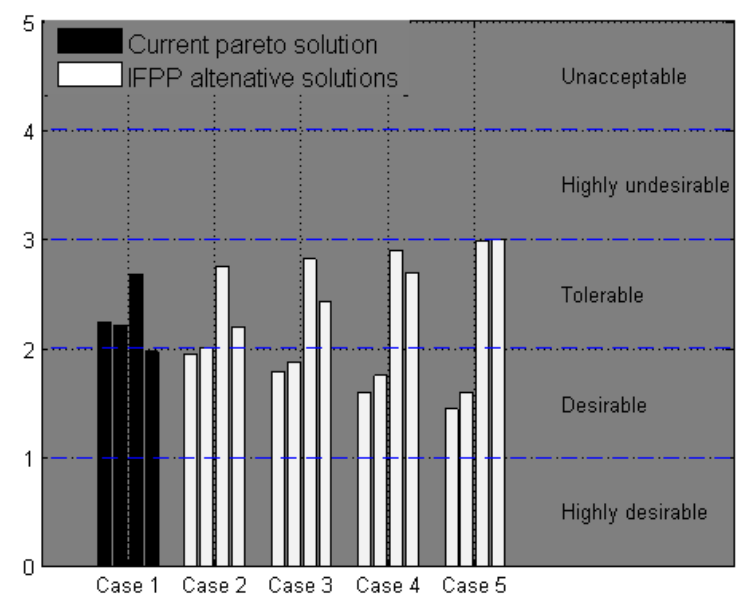

Fig. 17: Alternative solutions for Scenario 2 generated by IFPP

applying this strategy, the time history with respect to the state and control variables can be much smoother. This can be seen from Figs.12-16, where the distribution of grid points tends to be denser at those areas having a high value of curvature. On the other hand, the distribution of the temporal points tends to be sparser at those flat areas. Therefore, compared with other direct transcription methods, the proposed strategy is effective in terms of capturing discontinuity in the control derivatives after each iteration. Another main advantage of using the proposed mesh refinement strategy is that it can control the size of resulting nonlinear programming problem by fixing the number of subintervals and grid points. Since the current onboard computational devices may not be able to satisfy the requirement of the large computational power and time needed for solving large scale NLP problem, it is potentially useful for the adaptive multiple shooting method in terms of real-time applications.

As a result, all the figures and data provided earlier confirm the feasibility of the proposed adaptive multiple shooting method and IFPP strategy. By applying the combined algorithm, the SMV can reach the target position without violating three path constraints and boundary conditions. Moreover, the decision maker can specify his/her preferences in different objective functions during the optimization process and get new solutions, which can satisfy his/her new demands.

\section{CONCLUSION}

In this work, in order to design a trajectory which can achieve multiple goals for the SMV, the single objective optimization formulation was extended to multi-objective optimization, including minimum final time, aerodynamic heating, maximum final velocity and mass. Considering the fuzzy characteristic of evaluating objectives, an interactive fuzzy physical programming approach is constructed to generate the compromised solution based on the decision maker's preference. For a decision maker, there is no need to specify the weight information. Alternatively, this can be achieved by specifying the boundary aspirations of each preference region. Simulation results indicated the highly correlated and conflicting relationships between the objectives investigated in this paper. A compromised solution, where all the objective values can in the acceptable region, is found via the proposed method. Moreover, by applying the fuzzy physical programming based interactive strategy, two decision-making scenarios can be achieved successfully. This further confirms the effectiveness of the proposed multi-objective method for solving the SMV trajectory planning problem.

\section{REFERENCES}

[1] P. Ghiglino, J. L. Forshaw, and V. J. Lappas, “Oqtal: Optimal quaternion tracking using attitude error linearization," IEEE Transactions on Aerospace and Electronic Systems, vol. 51, no. 4, pp. 2715-2731, 2015.

[2] H. Gui, G. Vukovich, and S. Xu, "Attitude tracking of a rigid spacecraft using two internal torques," IEEE Transactions on Aerospace and Electronic Systems, vol. 51, no. 4, pp. 2900-2913, 2015. 
[3] S. R. Kumar and D. Ghose, "Impact time guidance for large heading errors using sliding mode control," IEEE Transactions on Aerospace and Electronic Systems, vol. 51, no. 4, pp. 3123-3138, 2015.

[4] J. Zhang, K. Ma, G. Meng, and S. Tian, "Spacecraft maneuvers via singularity-avoidance of control moment gyros based on dual-mode model predictive control," IEEE Transactions on Aerospace and Electronic Systems, vol. 51, no. 4, pp. 2546-2559, 2015.

[5] R. Jeremy, Launch Vehicle Trajectory Optimization Using a Legendre Pseudospectral Method, ser. Guidance, Navigation, and Control and Co-located Conferences. American Institute of Aeronautics and Astronautics, 2003, doi:10.2514/6.2003-5640.

[6] S. Yuri, H. Charles, and J. Mark, Reusable launch vehicle control in multiple time scale sliding modes, ser. Guidance, Navigation, and Control and Co-located Conferences. American Institute of Aeronautics and Astronautics, 2000.

[7] H. Duan and S. Li, "Artificial bee colony based direct collocation for reentry trajectory optimization of hypersonic vehicle," IEEE Transactions on Aerospace and Electronic Systems, vol. 51, no. 1, pp. 615-626, 2015.

[8] Z. Duan and X. R. Li, "Analysis, design, and estimation of linear equality-constrained dynamic systems," IEEE Transactions on Aerospace and Electronic Systems, vol. 51, no. 4, pp. 2732-2746, 2015.

[9] C. W. Brunner and P. Lu, "Skip entry trajectory planning and guidance," Journal of Guidance, Control, and Dynamics, vol. 31, no. 5, pp. 1210-1219, 2008.

[10] A. L. Rodriquez-Vazquez, M. A. Martin-Prats, and F. Bernelli-Zazzera, "Spacecraft magnetic attitude control using approximating sequence riccati equations," IEEE Transactions on Aerospace and Electronic Systems, vol. 51, no. 4, pp. 3374-3385, 2015.

[11] D. A. Benson, G. T. Huntington, T. P. Thorvaldsen, and A. V. Rao, "Direct trajectory optimization and costate estimation via an orthogonal collocation method," Journal of Guidance, Control, and Dynamics, vol. 29, no. 6, pp. 1435-1440, 2006.

[12] J. T. Betts, "Survey of numerical methods for trajectory optimization," Journal of Guidance, Control, and Dynamics, vol. 21, no. 2, pp. 193-207, 1998.

[13] C. L. Darby, W. W. Hager, and A. V. Rao, "Direct trajectory optimization using a variable low-order adaptive pseudospectral method," Journal of Spacecraft and Rockets, vol. 48, no. 3, pp. 433-445, 2011.

[14] X. Liu and P. Lu, "Solving nonconvex optimal control problems by convex optimization," Journal of Guidance, Control, and Dynamics, vol. 37, no. 3, pp. 750-765, 2014.

[15] J. Timothy, S. Christopher, F. Franklin, and R. Anil, Constrained Trajectory Optimization Using Pseudospectral Methods, ser. Guidance, Navigation, and Control and Co-located Conferences. American Institute of Aeronautics and Astronautics, 2008, doi:10.2514/6.2008-6218.

[16] J. T. Betts, Practical Methods for Optimal Control and Estimation Using Nonlinear Programming. Cambridge University Press, 2009.

[17] J. T. Betts and W. P. Huffman, "Mesh refinement in direct transcription methods for optimal control," Optimal Control Applications and Methods, vol. 19, no. 1, pp. 1-21, 1998.

[18] Q. Gong, F. Fahroo, and I. M. Ross, "Spectral algorithm for pseudospectral methods in optimal control," Journal of Guidance, Control, and Dynamics, vol. 31, no. 3, pp. 460-471, 2008.

[19] C.-H. Huang, J. Galuski, and C. L. Bloebaum, "Multi-objective pareto concurrent subspace optimization for multidisciplinary design," AIAA Journal, vol. 45, no. 8, pp. 1894-1906, 2007.

[20] A. Messac, "Physical programming - effective optimization for computational design," AIAA Journal, vol. 34, no. 1, pp. 149-158, 1996.

[21] R. V. Tappeta, J. E. Renaud, A. Messac, and G. J. Sundararaj, "Interactive physical programming: Tradeoff analysis and decision making in multicriteria optimization,” AIAA Journal, vol. 38, no. 5, pp. 917-926, 2000.

[22] P. Shi, X. Su, and F. Li, "Dissipativity-based filtering for fuzzy switched systems with stochastic perturbation," IEEE Transactions on Automatic Control, vol. 61, no. 6, pp. 1694-1699, 2016.

[23] P. Shi, Y. Zhang, M. Chadli, and R. K. Agarwal, "Mixed h-infinity and passive filtering for discrete fuzzy neural networks with stochastic jumps and time delays," IEEE Transactions on Neural Networks and Learning Systems, vol. 27, no. 4, pp. 903-909, 2016.

[24] K. Deb, A. Pratap, S. Agarwal, and T. Meyarivan, "A fast and elitist multiobjective genetic algorithm: Nsga-ii," IEEE Transactions on Evolutionary Computation, vol. 6, no. 2, pp. 182-197, 2002.

[25] M. Burger and W. Muhlhuber, "Numerical approximation of an sqp-type method for parameter identification," SIAM Journal on Numerical Analysis, vol. 40, no. 5, pp. 1775-1797, 2002.

[26] M. Heinkenschloss and D. Ridzal, "A matrix-free trust-region sqp method for equality constrained optimization," SIAM Journal on Optimization, vol. 24, no. 3, pp. 1507-1541, 2014.

[27] J. Laurent-Varin, F. Bonnans, N. Berend, M. Haddou, and C. Talbot, "Interior-point approach to trajectory optimization," Journal of Guidance, Control, and Dynamics, vol. 30, no. 5, pp. 1228-1238, 2007.

[28] J. Nocedal and S. J. Wright, "Numerical optimization, springer series in operations research," Siam J Optimization, 1999.

[29] X. R. Li and V. P. Jilkov, "Survey of maneuvering target tracking. part i. dynamic models," IEEE Transactions on Aerospace and Electronic Systems, vol. 39, no. 4, pp. 1333-1364, 2003.

[30] C. H. Lee, T. H. Kim, and M. J. Tahk, "Effects of time-to-go errors on performance of optimal guidance laws," IEEE Transactions on Aerospace and Electronic Systems, vol. 51, no. 4, pp. 3270-3281, 2015.

[31] P. Lu, "Predictor-corrector entry guidance for low-lifting vehicles," Journal of Guidance, Control, and Dynamics, vol. 31, no. 4, pp. 1067-1075, 2008.

[32] _ _ "Entry guidance using time-scale separation in gliding dynamics," Journal of Spacecraft and Rockets, vol. 52, no. 4, pp. 1253-1258, 2015.

[33] Y. Zhao and P. Tsiotras, "Density functions for mesh refinement in numerical optimal control," Journal of Guidance, Control, and Dynamics, vol. 34, no. 1, pp. 271-277, 2011.

[34] J. Hugger, "The theory of density representation of finite element meshes. examples of density operators with quadrilateral elements in the mapped domain," Computer Methods in Applied Mechanics and Engineering, vol. 109, no. 1, pp. 17-39, 1993. 
[35] R. Chai, A. Savvaris, and A. Tsourdos, "Fuzzy physical programming for space manoeuvre vehicles trajectory optimization based on hp-adaptive pseudospectral method," Acta Astronautica, vol. 123, pp. 62-70, 2016.

[36] K.-P. Lin, Y.-Z. Luo, and G.-J. Tang, "Multi-objective optimization of space station logistics strategies using physical programming," Engineering Optimization, vol. 47, no. 8, pp. 1140-1155, 2015.

[37] F. de Oliveira, N. M. P. Volpi, and C. R. Sanquetta, "Goal programming in a planning problem," Applied Mathematics and Computation, vol. 140, no. 1, pp. $165-178,2003$.

[38] L.-H. Chen and F.-C. Tsai, "Fuzzy goal programming with different importance and priorities," European Journal of Operational Research, vol. 133, no. 3, pp. 548-556, 2001. 


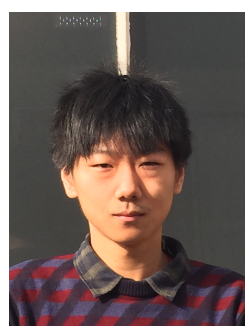

Runqi Chai was born in Beijing, China, in 1993. He is currently pursuing the Ph.D. degree in Aerospace Engineering at Cranfield University. His research interests include trajectory optimization, guidance and control.

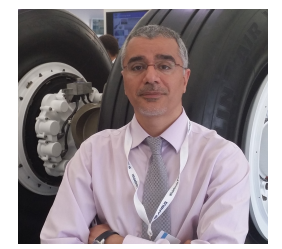

Al Savvaris is a Reader in the Centre for Cyber-Physical Systems at Cranfield University. He established the Autonomous Vehicle Dynamics and Control MSc course and the COMAC training programme at Cranfield. His research interests and activities include systems integration, hybrid energy management, communication systems, embedded systems, guidance and control. Currently he is working on InnovateUK funded AirStart and USMOOTH projects. In the past he worked on the FLAVIIR and ASTRAEA UAS projects, developing new technologies for unmanned systems; working on hardware and system integration. He participated in FP6, in the scope of the FLYSAFE project, working on next generation integrated safety systems. He was a member of the Autonomous Systems National Technical Committee; EPSRC College review member and reviewer on several international publications including IMechE and IEEE. He has published over 50 peer-reviewed journal and conference papers.

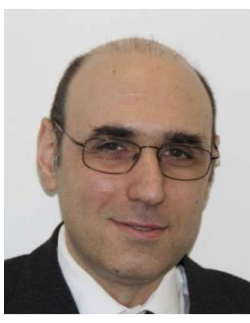

papers.

Antonios Tsourdos is a professor of control systems at Cranfield University. Appointed Head of the Autonomous Systems Group in 2007. Professor Tsourdos was member of the Team Stellar, the winning team for the UK MoD Grand Challenge (2008) and the IET Innovation Award (Category Team, 2009). Antonios is an editorial board member on several international publications including IMechE and IEEE. He is member if the IFAC Technical Committee on Intelligent Autonomous Vehicles, the IET Executive Team on Robotics and Automation and the ATI Autonomous Systems National Technical Committee. He was also involved in the SEAS DTC on Autonomous Systems Verifications. Professor Tsourdos has also been engaged in research on guidance \& control for single and multiple vehicles as well as verifiable autonomy of autonomous systems and lately dealing with the newly-important subjects of integrated system health management and cyber-physical systems. He has published over 100 peer-reviewed journal and conference

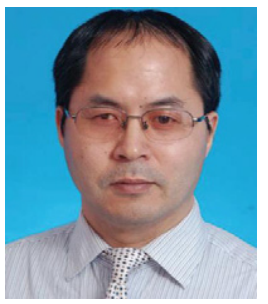

Yuanqing Xia was born in Anhui Province, China, in 1971. He received the B.S. degree from the Department of Mathematics, Chuzhou University, Chuzhou, China, in 1991, the M.S. degree in fundamental mathematics from Anhui University, Wuhu, China, in 1998, and the Ph.D. degree in control theory and control engineering from the Beijing University of Aeronautics and Astronautics, Beijing, China, in 2001.

His current research interests are in the fields of networked control systems, robust control and signal processing, active disturbance rejection control and flight control. He has published eight monographs with Springer and Wiley, and more than 100 papers in journals. He has obtained Second Award of the Beijing Municipal Science and Technology (No. 1) in 2010, Second National Award for Science and Technology (No. 2) in 2011, and Second Natural Science Award of The Ministry of Education (No. 1) in 2012. He is a Deputy Editor of the Journal of the Beijing Institute of Technology, Associate Editor of Acta Automatica Sinica, Control Theory and Applications, the International Journal of Innovative Computing, Information and Control, and the International Journal of Automation and Computing. 
Fig.1 General mission profile

Fig.2 Example of density function

Fig.3 Fuzzy preference function for Class 1-S

Fig.4 Interactive decision-making procedure

Fig.5 Pareto front obtained via NSGA-II

Fig.6 Pareto front obtained via NSGA-II

Fig.7 Pareto front obtained via NSGA-II

Fig.8 Single-objective solutions

Fig.9 Comparison between weight-sum and FPP

Fig.10 Comparison between GP, FGP and FPP

Fig.11 Alternative solutions for Scenario 1 generated by IFPP

Fig.12 Trajectories for altitude, speed, flight path angle and heading angle in five cases

Fig.13 Trajectories for mass and path constraints in five cases

Fig.14 Trajectories for actual AOA and demand AOA in five cases

Fig.15 Trajectories for actual bank angle and demand bank angle in five cases

Fig.16 Trajectories for actual thrust and demand thrust in five cases

Fig.17 Alternative solutions for Scenario 2 generated by IFPP

Table I Coefficients and maximum value of path constraints

Table II Control parameters of weight-sum and FPP methods 
2017-04-24

\section{An interactive fuzzy physical programming for solving multiobjective skip entry problem}

Chai, Runqi

IEEE

Chai R, Savvaris A, Tsourdos A, Xia Y, An interactive fuzzy physical programming for solving multiobjective skip entry problem, IEEE Transactions on Aerospace and Electronic Systems, Volume 53, Issue 5, Oct. 2017, pp2385-2398

http://dx.doi.org/10.1109/TAES.2017.2696281

Downloaded from Cranfield Library Services E-Repository 